\title{
Navigating Communication Between Western Medicine And Traditional Chinese Medicine: A Case Study of Cancer Patients In Hong Kong
}

Jack Pun ( $\square$ jack.pun@cityu.edu.hk)

City University of Hong Kong https://orcid.org/0000-0002-8043-7645

\section{Wendy Wong}

The Chinese University of Hong Kong https://orcid.org/0000-0001-8731-0498

\section{Research Article}

Keywords: Traditional Chinese Medicine (TCM), Cancer, Quality of Life, Hong Kong, Interpretative phenomenological analysis, Patients

Posted Date: December 2nd, 2021

DOl: https://doi.org/10.21203/rs.3.rs-1124302/v1

License: (c) (i) This work is licensed under a Creative Commons Attribution 4.0 International License.

Read Full License 


\section{Abstract}

Background: Traditional Chinese Medicine (TCM) is a growing phenomenon around the world. However, despite its historical role in Chinese society, there has been limited research exploring the nature of communication around cancer patients who receive TCM care in additional to conventional medicine. If TCM practitioners with adequate knowledge in understanding the needs and communication issues around patients with cancer, particularly those who simultaneously receive conventional medicine, it will lead to a better quality of care and result in better clinical outcomes such as high patient satisfaction and compliance with treatments.

Objectives: To fill in this gap, this study aims to explore the nature of the communication amongst cancer patients in Hong Kong who receive TCM treatment in addition to conventional medicine.

Methods: Twenty patients, 5 oncologists and 5 TCM practitioners were invited to conduct in-depth interviews to elicit their views towards their TCM treatment. A qualitative approach called Interpretative phenomenological analysis (IPA) is adopted.

Results: Based on the themes that emerged from the interview transcripts, we outline the priorities of communication when dealing with cancer patients who receive both TCM and conventional medicine. A framework was developed to assist training TCM practitioners to better integrate their patients' conventional medical history when delivering patient care.

Conclusion: The findings of this study can shed light to inform priorities in communication when caring for patients who opt for TCM care in addition to conventional treatments, and to provide useful information for developing future clinical research of providing integrated approach between TCM and conventional medicine in treating cancer patients.

\section{Background}

With a long and profound history of focusing on the patient's physical and interpersonal needs, Traditional Chinese Medicine (TCM) has always been attaching great importance to a patient-centred treatment. However, as time marches on, the importance of conventional medicine rises rapidly on the land where TCM used to dominate, especially in cancer treatments, making patients sometimes hard to decide which method they should turn to. In China, TCM is widely used in treating Chinese cancer patients [1],[2],[3]. When deciding to choose TCM for treating cancer, patients make it is a self-help process with their strong cultural grounding as they believe it is an optimal and safe process when comparing to conventional medicine, leading to long-term positive effects in their treatments. 1 In the Chinese context, many patients and their family members are aware of the limitations of cancer therapy which provides a strong motivation to opt for TCM, hoping to integrate TCM as a complementary therapy in addition to their conventional medicine, such as radiotherapy and chemotherapy [4],[5]. However, there is limited study on exploring the communication nature of patients who receive both TCM and conventional medicine or the priorities of their communication needs. Using cancer patients' interview 
data, this article aims to the nature of the communication of cancer patients in Hong Kong who receive TCM treatment in addition to conventional medicine.

Cancer is the number one killer in Hong Kong. In 2018, cancer claimed 14,446 lives, accounting for about one-third of the total deaths in the local population [6]. According to the government's statistics, the number of new cancers has increased at a rate of about $2.9 \%$ per year in the past decade, hitting a historical high of 31,468 in 2016. It is believed that the annual average number of new cases will increase by about $35 \%$ to more than 42,000 by 2030 . The five most common cancers in Hong Kong are colorectal, lung, breast, prostate and liver cancer, which comprised over half (58.1\%) of all the new cases [6]. In a large-scale study exploring cancer patients' attitudes towards the use TCM treatment, of a total 786 respondents, over half of them (57.1\%) used at least one form of Chinese medicine before, during or after the conventional therapy. Surprisingly, two-thirds of the respondents did not inform their oncologists about using TCM [7]. Over two-thirds of the participants (68.2\%) believed that integrated TCM and conventional medicine was effective.

This newly uprising phenomenon has thus naturally brought up the question of whether they could get along well with one another, particularly within the field of cancer treatment. Some may argue that the combination and integration of TCM and conventional medicine could bring better effects, as Yang suggested in his article, Chinese and conventional integrative medicine treatment of medium and advanced lung cancer has shown to improve patients' Quality of Life (QOL) and survival rate, normally with the ability to control tumour development in the short term [8]. However, if to compare the long-term effect of using conventional medicine combined with TCM, with conventional medicine on the prognosis of patients with lung cancer, the combined treatment group turns to have better cumulative survival, yet without a significant difference. Moreover, after evaluating the efficacy and side effects of combined Chinese drugs and chemotherapy in treating advanced non-small cell lung cancer, it is suggested that combining Chinese drugs and chemotherapy can enhance the short-term therapeutic efficacy in the treatment of Non-small Cell Lung Cancer (NSCLC) and prolong patients' median survival time, but show no evident impact on time to progression [2].

TCM has some unique treatments that differ enormously compared to those used in conventional medicine. In TCM, every patient condition is handled individually and such patient-tailored treatments have a millennia-old tradition in TCM. To start with, by identifying the imbalance in the pulse, the life force or energy, and organs via examination, TCM doctors focus more on the patient's background (both social and natural), taking a holistic view of the relationship between the patient's physical body and the environment, in hope to find some track their diseases onto some factors in their daily routine. By tracking patients' daily activities, a TCM doctor can take reference and construct connections between the symptoms with it, in the belief that disease is a result of an unhealthy lifestyle. Chinese herb medicine is often used in TCM to enhance patients' physical and emotional well-beings. It is highly prevalent among cancer patients to use it to reduce cancer therapy-induced toxicities [9],[10]. 
In contrast, conventional medicine doctors focus not as much on the patient's overall habitual behaviours, but more on identifying the primary clinical concerns and causes of illness which the patient is currently facing. Moreover, when it comes to diagnostic approaches adopted, TCM practitioners try to identify the human body by system discrimination and cybernetic approaches, which allow them to understand the patient's illness or 'Zheng'[11]. 'Zheng', as an outcome of all the patient's symptoms and discomforts like imbalances in the pulse, life force or energy and organs identified via examination, is a vital indicator of the patient's health for a TCM practitioner. 'Zheng', if to be divided into parts, consists of inspection, auscultation and olfaction, inquiring and palpation, which in Chinese is called 'Si-Zhen', the process in which a TCM practitioner evaluates and judges the illness of a patient before the final diagnosis. The process of obtaining the result from 'Zheng', thus, requires an overall and holistic observation of multiple body sensors and organs, as well as a concentrated analysis of the patient's personal experience [11].

Conventional medicine, on the other hand, relies heavily on the scientific observations of the symptoms of the patient and tries to narrow down and locate the exact cause of it. During such treatments, scientific data generated from physical examinations are highly respected, even though this means there's not as much mutual communication between the practitioner and patient as that of TCM. As TCM philosophy suggests, cancer results from a lack of balance between yin and yang (the balance that maintains health), or a disturbance of the smooth flow of 'qi' (vital energy), which then manifests as a group of syndromes in which there lacks harmony in the body-mind-environment network. To restore the balance, TCM focuses on mobilizing and activating the body's natural resources. By enhancing the patients' inner defences and restoring their normal patterns, it is thought to help defeat cancer and promote healing. The disruption of ying-yang balance (the balance that contributes to health maintenance) and/or the smooth flow of 'qi' (vital energy) are the reasons for cancer according to TCM philosophy. The disturbances will then be embodied as syndromes where there is a lack of harmony in the body-mind-environment network. The concentration of TCM cancer therapy is to focus on stimulating and mobilizing the body's natural resources to counteract the balance. By re-establishing normal patterns and enhancing the inner defences, it is believed that cancer can be defeated and healing can be promoted. 1 In cancer treatment, there are five useful Chinese medicine modalities such as using Chinese herbal medicine, acupuncture and moxibustion, therapeutic massage, qigong and Chinese dietary therapy which have been identified effective in cancer therapy $[4,5,7]$.

Besides, acupuncture has been provided strong empirical evidence that it can largely relieve patients' pain and acute vomiting situation in conventional cancer treatment.[12],[13] This study aims to explore the nature of communication among cancer patients in Hong Kong who receive TCM treatment in addition to conventional medicine. To support future researches with priorities in communication when caring for patients who opt for TCM care in addition to standard treatments, and an integrated approach between TCM and conventional medicine in treating cancer patients.

\section{Methods}




\section{Study design and aims}

A qualitative approach called Interpretative phenomenological analysis (IPA) is adopted in the research process, which investigates personal lived experiences of the patient's within the field of psychology [9]. As stated by Smith and Osborn[14] , IPA mainly focuses on personal experiences, together with a detailed exploration of how an individual understands and makes sense of them [10]. To adopt the analysis theory correctly, certain assumptions are made beforehand. First, one is constantly in engagement with the world actively and their experiences being reflected to cohere with it [15]. Furthermore, the investigation of experiences is an allegorical analysis by both the participants and the researcher. To make sense of how the participants understand their world, the researchers and the participants are to be involved in a dual interpretation process, which is called the 'double hermeneutic'. Researchers have to play an active role and try to comprehend how the participants are making sense of their world [15]. With its ability to adapt the inaugural approach and capacity to investigate the participants' complex lived experiences, IPA was thus selected as the analytical method for this study.

The interview conducted with all the cancer patients who currently received both cares from TCM and conventional medicine in treating their cancer condition. The interviews explored their perceptions of communication practices in navigating their communication around their treatments and attitudes towards TCM care. Specifically, these interview asked participants the following questions: 1 ) What is your view about TCM service in Hong Kong? 2) From your experience, what role do TCM play in treating cancer patients? 3) What kinds of factors are key when patients decide to receive TCM care in addition to their conventional medicine treatment? 4) Are there any advantages that TCM can provide more to patients than they receive from conventional medicine? 5) Is there any opportunity that TCM and conventional medicine can be integrated into treating cancer?

\section{Data Analysis}

IPA, as an idiographic means of an approach that is adopted in this study, analyses a tightly defined group of participants who serve as a specific point of view in that particular area of study. Twenty patients, 5 oncologists and 5 TCM practitioners were recruited. The participants of this study are all experienced TCM doctors with multiple years of practising curing intestine cancer, or patients who have been diagnosed with it. The interview transcripts are analysed by adopting the analytical stages suggested by Smith et al [15]. To begin with, an individual focus and descriptive level are attached with great importance, before the analysis is moved to a more mutual understanding of the clinical context in an interpretative level. The interview transcript was read line by line, with extra focus on what was being said about cancer treatments and integration of TCM and conventional medicine, which were also placed as initial comments. Exploratory comments with the researcher's interpretations concerning the participant's use of language and communication issues concerning the topic were also added to the transcript as a second priority. By making these exploratory comments without looking into other interview transcripts, the researchers can develop concise themes that keep on track with the participant's rhetoric. At last, Smith et al.'s techniques of subsumption and abstraction were adopted and employed to 
determine similar patterns among the themes, which lead to a series of superordinate themes for the participants [15].

\section{Ethics}

Ethics approval from the Joint Chinese University of Hong Kong - New Territories East Cluster Clinical Research Ethics Committee was obtained (Ref no. 2016.307).

\section{Results}

The interviewees expressed a series of opinions on TCM and allowed a thorough examination of their perceptions on communication practices during their treatments and TCM care. Four common themes were identified among the interviewed cancer patients: 1) Confidence in TCM care in cancer treatment; 2) The communication mode of TCM; 3) Advantages of TCM over conventional medicine, and 4) Integration of TCM and conventional medicine in cancer care.

\section{1) Confidence in TCM in cancer treatment}

TCM in Hong Kong is attracting more and more patients with references to the interviewees. When talking about the trends of using TCM in curing intestine cancer, one patient (Lei) explained the assurance factor is what made him stick with TCM for a long time. In fact, to a large extent, people turn to TCM for mental assurance and confirmation due to their preferences of the TCM consultation style that involves the overall lifestyle and health condition of a patient before making a diagnosis. Patients would often be in favour of such detailed and tailored treatment, resulting in greater confidence in involving TCM in their cancer treatment [16],[17].

'If the practitioner could make you confident about what he's doing and lend you plenty of information about your health condition, it will make your heart sort of settled... In my case, since I have only visited TCM doctors twice, in overall speaking the experience has been quite positive, as I would get clear answers to whatever I asked. So far speaking I am quite satisfied with it.'

(Lei, interview 39).

Besides, TCM doesn't exclude other treatments at the same time or even encourage its patients to ask for other help if necessary. This is crucially important in clarifying the doubts that patients may have during a hard time, according to a cancer patient (Zung)'s interview. As TCM also recommends other treatment when needed, it gives patients a feeling that their health is of the utmost priority in TCM practitioners' eyes, thus making them willing to receive the information and suggestions provided by a TCM practitioner in the belief that it's right.

I have been consulting TCM for a long time since 2013, with conventional medicine simultaneously. I began to consult TCM even before I receive chemo. It was my sister who recommended Dr CHAN to me whom I kept visiting until he retired... The consultation between me and a TCM practitioner is not as 
frequent as that with a conventional medicine doctor, however, after listening to the explanations of a TCM practitioner, I got more confident even sometimes conventional medicine tells me the contrary. That's also the reason why I haven't stopped visiting TCM till now.'

\section{(Zung, interview 37).}

\section{2) The communication mode of TCM}

During a treatment process, it is inevitable for a patient to be scared and full of doubts about what's to come. In such conditions, the communication mode adopted by TCM, which focuses on learning about the general health conditions of a patient, would naturally build a connection between the practitioner and the patient. 5 With the foundation laid in the first place, their consultations could go much deeper than that in a conventional medicine context and make the patients calm as in the case of Mr MAN.

'I have visited TCM even before I visited a conventional medicine doctor... When the hospital informed me that I needed to receive chemo, I turned to my TCM practitioner for advice. He told me that I must go through the chemo process and I listened. He said that he understood it is hard to go through chemo and explained to me that it doesn't have any side effect... When I am visiting a TCM doctor, the whole consultation process is much more detailed, I would be asked for a report and he kept things down carefully to give me suggestions as well as the whole situation, which makes me feel so assured.'

(Man, interview 34).

On the other hand, because of the lack of general understanding among patients about the practice of TCM, this communication mode also faces its drawbacks. As Mr NG mentioned in his speech, it would be really hard to understand what's fundamentally going on if one hasn't learned the basics of a TCM treatment, unlike in the conventional medicine context where people tend to know about the basics more or less. This, however, doesn't seem to affect the understandings of the patient during a consultation. Like Ms GAM explained, the elaborations given by the TCM practitioner are clear and straightforward and such a communication mode adopted by TCM still works for them to make them assured.

'During the whole treatment process, the explanations of the practitioner were quite clear. Even though we didn't get to be told what functions the medicines hold, we didn't need to know that. All we think is that it helped us after operations.'(Gam, interview 35).

'Whenever I visit a TCM practitioner, I always feel the consultation is much more personal and well connected since it is always the same doctor I see when I am in a TCM consultation, unlike in conventional medicine ones, where I am appointed to different doctors who tend to finish the conversation within several minutes. It is assuring to know that someone keeps tracking your profile and help to control your illness. However, if you didn't know anything about TCM previously, what a TCM practitioner says to you may not make much sense. In that case, it would be conventional medicine that could offer clearer explanations.' 
(므, interview 33).

\section{3) Advantages of TCM over conventional medicine}

To start with, Leung brought up TCM's suitability to patients, which is lacked in conventional medicine. By suitability he explained that no matter it's a cold or emotion fluctuation, TCM always finds a way to combine the overall discomforts, while conventional medicine turns to separate those in several different branches and treat various illnesses individually. Moreover, Leung mentioned that TCM turns to be combined with the weather and environmental conditions while healing, putting more attention to the patients themselves. While on the contrary, conventional medicine treats their patients more like a task, by which he also hopes for an improvement in this situation.

"The change of seasons leads to different causes to the cold, such as wind, cold, heat, wetness, dryness, and fire. There are different methods to treat cold with different causes. We also have to take the seven emotions caused by the cold into account before prescribing TCM to patients. These emotions are happiness, anger, worry, thoughtful, scare, horror, and sadness."

(Leung, interview 5).

The result of conventional treatments, he claimed, could be ineffective to patients sometimes. He met a patient who fainted after eating the prescribed convention medicine.

"Sometimes conventional medicine doctors randomly give patients medicine without considering their bodies thoroughly. One of my patients fainted after eating conventional medicine and came to find me immediately."

\section{(Leung, interview 5).}

However, He said he could not blame conventional medicine doctors as they have their way of treating patients, just like TCM doctors. Both TCM and conventional medicine doctors are healthcare practitioners who put their patients' health to the highest level of their concern. The same suggestion is then verified by a patient diagnosed with intestine cancer with such statements below:

'It can't promise to cure you, but you could visit TCM doctors. And you should visit a TCM doctor after visiting a conventional medicine doctor because it will help you gain a stronger body system, as the TCM treatments tend to penetrate your body and bring some fundamental changes. conventional medicine, on the other hand, doesn't provide this function.'

(Lai, interview 36).

\section{4) Integration of TCM and conventional medicine in cancer care}

TCM could not be used in isolation when dealing with cancer, especially when it comes to the first stage of diagnosing. Furthermore, with different focuses during the treatment process, TCM and conventional 
medicine could provide the patient with various benefits that could not be compensated by using another [18].

In my perception, when talking about TCM in cancer treatment, it has to be diagnosed using conventional medicine, and analysed further by TCM, with these two combined. The reason is that you can only rely on conventional medicine to make sure it is cancer, the same as you can only use the differentiation theory in TCM to take the medicines properly.'

(Chen, interview 1).

When talking about the attitudes that are held by many doctors and the general public, Dr MA mentioned that he has noticed a constant rivalry and quarrel between TCM and conventional medicine, arguing which should dominate the treatment. However, he also thinks that both sides should treat each other with respect and more understanding, based on more in-depth communications in between.

'And I hope you could take this as a remark, that is I think TCM could have a much deeper dialogue with conventional medicine, with everybody prejudicing less against one another. I think I am pretty neutral when talking about the relationship between TCM and conventional medicine, as my family are traditional doctors in TCM and I am a conventional medicine doctor. Whenever I see conflicts existing between the two sides, I always think they could coexist in harmony.'

(Ma, interview 12).

\section{Discussion}

By exploring the communication mode between cancer patients and their doctors in the TCM context, this project is believed to have contributed to the existing literature concerning the doctor-patient communication and ways of improving patient confidence and satisfaction in Hong Kong. Through comparing the different approaches adopted in conventional medicine and TCM consultations, it is concluded that TCM practitioners exchange medical information with patients in ways that allow them to construct an interpersonal relationship with their patients, which leads to better clinical outcomes and higher patient satisfaction as a result. The results of this study correspond to the existing literature which explores the nature of doctor-patient interaction in Hong Kong, that healthcare providers' use of effective, interpersonally linguistic communication strategies will lead to more positive patient outcomes. Consequently, it is concluded that when treating cancer patients who are consulting conventional medicine and TCM at the same time, healthcare providers in TCM context are more prone to target their communication effectively and balances interpersonal communication with the communication of medical expertise that is integral to ensuring patients' involvements, comprehension and satisfaction of their care. These linguistic strategies adopted by most TCM practitioners, which are also necessary to achieve effective patient-centred care, should be promoted in the training of future TCM practitioners to help these TCM practitioners to identify effective communication strategies in dealing with Cantonese patients, making conventional medicine and TCM well integrated. The findings of this project can also 
extend our understandings of communications with cancer patients whose primary needs, as the results suggest, are to be rested assured. Furthermore, the study also provides a firm evidence-based foundation for the development of communication strategies and interventions to improve practice, suggesting multiple benefits for conventional medicine doctors and caregivers to communicate with patients in using TCM terminology. However, a drawback of this study is the number of correspondents it receives, considering the small group of people who have visited a TCM practitioner in reality.

\section{Conclusion}

The role of the TCM practitioner is not only of investigating the causes of a patient's concern and treating them but also of exploring the overall conditions that can allow a mutual understanding of the patient's problems. As differences between the patient's and practitioner's frames for concerns may lead to inappropriate diagnosis and noncompliance to treatment, resulting in medical failures, it is vitally important to understand the communication methods adopted during a consultation. Comparing the different modes of communication between TCM and conventional medicine, despite they are rather different in terms of philosophy (conventional medicine is based on scientific data while TCM is based on experience, language (terminology of medicine and disease) and consultation procedures, there is a trend in combining both TCM and conventional medicine in treating patients as the study has found out. When treating patients that are consulting TCM and conventional medicine simultaneously, TCM practitioners are more skilled at soothing their moods and explaining the overall conditions of their health, thus significantly improving the quality of health care, despite the increasing number of patient presentations, greater cultural and linguistic diversity and patients with both multiple morbidities and a wide variety of conditions. In particular, the utilization of natural communication skills in TCM consultation can lead to greater patients' participation and involvement, which could also be exampled as to suggest a better way of integrating TCM and conventional medicine in treating cancers.

\section{Declarations}

\section{Acknowledgement}

The authors would like to thank all the patients and staff members involved in this study.

\section{Authors' Contributions}

J.P. and W.W. contributed to the design of the study, data collection, data analysis and interpretation. J. Pun drafted the article and both authors made a final approval of the submitted version.

\section{Funding}

The authors received no financial support for the research, authorship and/or publication of this article.

\section{Availability of data and materials:}


Not applicable.

\section{Declarations}

\section{Ethics approval}

Ethics approval from the Joint Chinese University of Hong Kong - New Territories East Cluster Clinical Research Ethics Committee was obtained (Ref no. 2016.307).

\section{Competing interests}

The authors declare that they have no competing interests.

\section{Patient and Public Involvement}

Patients and/or the public were not involved in the design, or conduct, or reporting, or dissemination plans of this research.

\section{References}

[1]Xu W, Towers AD, Li P, COLLET JP. Traditional Chinese medicine in cancer care: perspectives and experiences of patients and professionals in China. European journal of cancer care. 2006 Sep;15(4):397403.

[2] Chen Z, Gu K, Zheng Y, Zheng W, Lu W, Shu XO. The use of complementary and alternative medicine among Chinese women with breast cancer. The Journal of Alternative and Complementary Medicine. 2008 Oct 1;14(8):1049-55.

[3] Chen B, Bernard A, Cottrell R. Differences between family physicians and patients in their knowledge and attitudes regarding traditional Chinese medicine. Integrative Medicine. 2000 Mar 1;2(2-3):45-55.

[4] Adams M, Jewell AP. The use of complementary and alternative medicine by cancer patients. international Seminars in Surgical Oncology 2007 Dec (Vol. 4, No. 1, pp. 1-7). BioMed Central.

[5] Liu CH, Tang WR, Wang HM, Lee KC. How cancer patients build trust in traditional Chinese medicine. European Journal of Integrative Medicine. 2013 Dec 1;5(6):495-500.

[6] The Government of the Hong Kong Special Administrative Region. Hong Kong Cancer Strategy 2019. 2019 July. Available from https://www.chp.gov.hk/files/pdf/aw_summary_web.pdf

[7] Lam YC, Cheng CW, Peng H, Law CK, Huang X, Bian Z. Cancer patients' attitudes towards Chinese medicine: a Hong Kong survey. Chinese Medicine. 2009 Dec 1;4(1):25.

[8] Yang S, Cui M, Li HY, Zhao YK, Gao YH, Zhu HY. A meta-analysis of the effectiveness of Chinese and Western integrative medicine on medium and advanced lung cancer. Chinese journal of integrative 
medicine. 2012 Nov 1;18(11):862-7.

[9] Liu TG, Xiong SQ, Yan Y, Zhu H, Yi C. Use of Chinese herb medicine in cancer patients: a survey in southwestern china. Evidence-Based Complementary and Alternative Medicine. 2012 Oct;2012.

[10] Carmady B, Smith CA. Use of Chinese medicine by cancer patients: a review of surveys. Chinese Medicine. 2011 Dec 1;6(1):22.

[11] Lu AP, Jia HW, Xiao C, Lu QP. Theory of traditional Chinese medicine and therapeutic method of diseases. World journal of gastroenterology: WJG. 2004 Jul 1;10(13):1854.

[12] Pan CX, Morrison RS, Ness J, Fugh-Berman A, Leipzig RM. Complementary and alternative medicine in the management of pain, dyspnea, and nausea and vomiting near the end of life: a systematic review. Journal of Pain and symptom management. 2000 Nov 1;20(5):374-87.

[13] Macek C. East meets West to balance immunologic yin and yang. Jama. 1984 Jan 27;251(4):433-9.

[14] Smith JA \& Osborn M. Interpretative phenomenological analysis. Qualitative psychology: A practical guide to research methods. 2008:53-80.

[15] Smith JA, Flowers P, \& Osborn M. Interpretative phenomenological analysis and the psychology of health and illness. In Material discourses of health and illness. Routledge. 2013:77-100.

[16] Lam TP. Strengths and weaknesses of traditional Chinese medicine and Western medicine in the eyes of some Hong Kong Chinese. Journal of Epidemiology \& Community Health. 2001 Oct 1;55(10):762-5.

[17] Chung VC, Lau CH, Wong EM, Yeoh EK, Griffiths SM. Perceived quality of communication amongst outpatients in western and traditional Chinese medicine clinics in a Chinese population. Health policy. 2009 Apr 1;90(1):66-72.

[18] Konkimalla VB, Efferth T. Evidence-based Chinese medicine for cancer therapy. Journal of ethnopharmacology. 2008 Mar 5;116(2):207-10. 